\title{
The Be K-edge in beryllium oxide and chalcogenides: soft $x$-ray absorption spectra from first-principles theory and experiment
}

Weine Olovsson, L Weinhardt, O Fuchs, I Tanaka, P Puschnig, E Umbach, C Heske and C Draxl

\section{Linköping University Post Print}

\section{Tweet}

N.B.: When citing this work, cite the original article.

Original Publication:

Weine Olovsson, L Weinhardt, O Fuchs, I Tanaka, P Puschnig, E Umbach, C Heske and C Draxl, The Be K-edge in beryllium oxide and chalcogenides: soft X-ray absorption spectra from first-principles theory and experiment, 2013, Journal of Physics: Condensed Matter, (25), 31.

http://dx.doi.org/10.1088/0953-8984/25/31/315501

Copyright: Institute of Physics: Hybrid Open Access http://www.iop.org/

Postprint available at: Linköping University Electronic Press http://urn.kb.se/resolve?urn=urn:nbn:se:liu:diva-97251 


\title{
The Be K-edge in beryllium oxide and chalcogenides: soft x-ray absorption spectra from first-principles theory and experiment
}

\author{
W. Olovsson ${ }^{1,2,3}$, L. Weinhardt ${ }^{4,5,6}$, O. Fuchs ${ }^{4}$, I. Tanaka ${ }^{3,7}$, P. \\ Puschnig $^{2,8}$, E. Umbach ${ }^{4,5}$, C. Heske ${ }^{4,5,6}$ and C. Draxl ${ }^{2,9}$ \\ 1 Department of Physics, Chemistry and Biology (IFM), Linköping University, \\ SE-581 83 Linköping, Sweden \\ ${ }^{2}$ Chair of Atomistic Modelling and Design of Materials, Montanuniversität Leoben, \\ Franz-Josef-Straße 18, A-8700 Leoben, Austria \\ 3 Department of Materials Science and Engineering, Kyoto University, Sakyo, Kyoto \\ 606-8501, Japan \\ ${ }^{4}$ Experimentelle Physik II, Universität Würzburg, Am Hubland, 97074 Würzburg, \\ Germany \\ ${ }^{5}$ Karlsruhe Institute of Technology, D-76021 Karlsruhe, Germany \\ ${ }^{6}$ Department of Chemistry, University of Nevada, Las Vegas, 4505 Maryland Pkwy, \\ Las Vegas, NV 89154-4003 \\ 7 Nanostructures Research Laboratory, Japan Fine Ceramics Centre, Atsuta, Nagoya \\ 456-8587, Japan \\ 8 Institute of Physics, University of Graz, 8010 Graz, Austria \\ 9 Department of Physics and IRIS Adlershof, Humboldt-Universität zu Berlin, Zum \\ Großen Windkanal 6, D-12489 Berlin, Germany \\ E-mail: weine.olovsson@ifm.liu.se
}

\begin{abstract}
We have carried out a theoretical and experimental investigation of the beryllium K-edge soft x-ray absorption fine structure of beryllium compounds in the oxygen group, considering $\mathrm{BeO}, \mathrm{BeS}, \mathrm{BeSe}$, and BeTe. Theoretical spectra are obtained $a b$ initio, through many-body perturbation theory, by solving the Bethe-Salpeter equation (BSE), and by supercell calculations using the core-hole approximation. All calculations are performed with the full-potential linearized augmented plane-wave method. It is found that the two different theoretical approaches produce a similar fine structure, in good agreement with the experimental data. Using the BSE results, we interpret the spectra, distinguishing between bound core excitons and higher energy excitations.
\end{abstract}




\section{Introduction}

Beryllium oxide has a very high thermal conductivity and electrical resistivity, and is among the hardest known materials, making it relevant for many practical applications, for example as a refractory and heat-sink material. Beryllium chalcogenides are of interest, for instance, for improving the lifetime of optoelectronic II-VI semiconductor devices [1]. To shed light on the electronic structure of these materials, we report in this paper on an investigation of the beryllium $1 s$ near-edge soft x-ray absorption fine structure (NEXAFS) spectra. We consider the wide band-gap insulator BeO and the semiconducting chalcogenides BeS, BeSe, and BeTe. At ambient conditions, BeO forms in the hexagonal wurtzite structure, while BeS, BeSe, and BeTe adopt the cubic zincblende lattice.

NEXAFS, or x-ray absorption spectroscopy (XAS), is a widespread method for characterizing the electronic and chemical properties of materials [2,3]. The resulting absorption spectrum is element-specific, sensitive to the local chemical environment of the probed atom, and gives insights into the unoccupied electronic valence states. Especially in combination with theoretical analysis, it can be used to gain deep insight into the electronic structure of the investigated material.

In this work, we apply two different first-principles techniques to obtain the theoretical spectra. First, we solve the equation of motion for the electron-hole twoparticle Green's function, the Bethe-Salpeter equation (BSE) [4, 5, 6, 7, 8, 9, 10] of many-body perturbation theory. Second, we perform supercell calculations within density functional theory (DFT) $[11,12]$. Here we apply the commonly used final-state rule (FSR) [13] to obtain the X-ray absorption spectra. In practice, we insert a corehole corresponding to the core-edge at a specific atom and add a screening electron into the valence to ensure charge neutrality. The system is thereafter calculated selfconsistently. Furthermore, we monitor the effect on the spectral shape by applying the Slater transition state [14], by reducing the core-hole to half. There are many different implementations for XAS in the literature, see for example [3, 15, 16, 17, 18]. FSR is also frequently used in connection with X-ray emission and photoelectron spectroscopy. For applications to excitation energies and core-level binding energy shifts it is closely connected to $\Delta$-self-consistent-field $(\triangle \mathrm{SCF})$ calculations. In $\Delta \mathrm{SCF}$ calculations the total energy difference between systems in their respective initial (unperturbed) and final (perturbed) state is utilized, see for example $[19,20,21,22]$. In this work we do not attempt to compute the energy position of the core-edges, instead the different theoretical and experimental spectra are directly aligned for easy comparison with each other. The BSE and supercell calculation methods described above account for the core-hole effect via different routes. Note that FSR and BSE are expected to produce similar spectra for the deeper core-region, as discussed in [23]. For all calculations, we employ the highly accurate all-electron full-potential linearized augmented plane-wave (FPLAPW) method [24]. The BSE calculations are performed with exciting [25, 26], which is an open-source package, while the supercell results are obtained by the WIEN2k 
code [27]. Experiments are carried out for BeO and BeS samples. Measurements for BeSe and BeTe were obtained previously, though only a range of $\sim 10 \mathrm{eV}$ from the edge of the spectra were presented [28].

Many studies, both experimental and theoretical, have been carried out for $\mathrm{BeO}$ (for recent work see, e.g. [29, 30, 31, 32]). The Be $1 s$ edge was investigated by Soininen et al. [33], using a pseudopotential-based BSE technique [34, 35] and, more recently, by pseudopotential (PP) supercell calculations within the core-hole approximation [36, 37]. Regarding the beryllium chalcogenides, there are several theoretical studies on their properties in the literature, see e.g. [38, 39, 40]. However, only a few experimental XAS studies were performed. As far as we know, no experimental data has yet been published on the electronic structure of BeS. One reason for this is that BeS is very difficult to obtain: At the time of the experiment, it was commercially available only in powder form. This means that the commonly established method to derive the electronic band structure, photoelectron spectroscopy, cannot be applied due to the lack of crystallite orientation (in addition to severe charging effects).

In the following, we will provide the theoretical and experimental background of our investigations. Thereafter, in section 4, we will present our findings and compare between the different theoretical approaches and experiments. Finally, a discussion and conclusions will be given in section 5 .

\section{Theory}

The present calculations are all performed from first-principles, utilizing the fullpotential linear augmented plane-wave method [24] within density-functional theory (DFT) [11, 12]. The Kohn-Sham states are obtained self-consistently and serve as a starting point for the subsequent solution of the Bethe-Salpeter equation of many-body perturbation theory $[4,5,6,7,8,9,10]$. These calculations are carried out with the opensource code exciting $[25,26]$. Below, a brief outline of the methodology is presented. For further details regarding theory and implementations with the FPLAPW basis, we refer to [10] (and references therein) and to [26, 41], respectively.

The eigenstates of the electron $(e)$ - hole $(h)$ pairs are obtained by solving the Bethe-Salpeter equation in terms of a matrix eigenvalue problem,

$$
\sum_{h^{\prime} e^{\prime} \mathbf{k}^{\prime}} H_{h e \mathbf{k}, h^{\prime} e^{\prime} \mathbf{k}^{\prime}}^{2 \mathrm{p}} A_{h^{\prime} e^{\prime} \mathbf{k}^{\prime}}^{\lambda}=E^{\lambda} A_{h e \mathbf{k}}^{\lambda},
$$

where a specific excitation $\lambda$ is associated with the eigenenergy $E^{\lambda}$ and eigenvector $A_{h e \mathbf{k}}^{\lambda}$. By disregarding spin-orbit coupling and considering only spin-singlet solutions, the effective Hamiltonian is given by a sum over three terms, $H^{2 \mathrm{p}}=H^{\mathrm{diag}}+H^{\mathrm{dir}}+2 H^{\mathrm{x}}$. Thereby, the diagonal term, $H^{\text {diag }}$, is represented by the eigenenergy differences between the $e$ and $h$ states. The direct term, $H^{\text {dir }}$, contains the statically screened Coulomb interaction, which is attractive and responsible for bound core-excitons. The exchange term, $H^{\mathrm{x}}$, includes the short-range part of the unscreened Coulomb potential. By dropping the direct term in $H^{2 \mathrm{p}}$, one obtains the random-phase approximation (RPA) 
that includes local field effects. Also leaving out $H^{\mathrm{x}}$ corresponds to the independentparticle approximation (IPA).

After solving the BSE by diagonalizing the matrix in (1), the rate of photon absorption is given by the expression for the imaginary part of the dielectric function,

$$
\epsilon_{2}(\omega) \propto \sum_{\lambda}\left|\sum_{h e \mathbf{k}} A_{h e \mathbf{k}}^{\lambda} \frac{\langle h \mathbf{k}|p| e \mathbf{k}\rangle}{\varepsilon_{e \mathbf{k}}-\varepsilon_{h \mathbf{k}}}\right|^{2} \times \delta\left(E^{\lambda}-\omega\right),
$$

where $\varepsilon$ are eigenenergies and $\langle h \mathbf{k}|p| e \mathbf{k}\rangle$ correspond to the momentum matrix elements. Using the electric dipole approximation $(\Delta l= \pm 1)$, only contributions from $p$-like orbitals are allowed for the K-edge $(1 s)$.

In practice, we use an $8 \times 8 \times 8 \mathbf{k}$ mesh in the case of $\mathrm{BeO}$, and a $10 \times 10 \times 10$ grid for the beryllium chalcogenides. The range of included conduction bands is 20 for $\mathrm{BeO}$ and 25 for the other compounds. These calculations are performed with the exciting code $[25,26]$.

Our second theoretical method is the supercell core-hole approach, utilizing the final state rule, for which we employ the WIEN2k program package [27]. In this case, a corehole is explicitly introduced in the $1 s$-orbital at a selected beryllium site. Thereafter, the system is fully relaxed by self-consistent calculations and finally Fermi's golden rule is applied to obtain the spectra, see e.g. [18]. The supercell's charge neutrality is conserved by promoting the electron into the conduction band. It is important that the supercell is of sufficient size to avoid spurious interaction between ionized atoms through the periodic boundary conditions. We thus have chosen cells of 108 atoms for wurtzite $\mathrm{BeO}$ and 128 atoms for the zinc-blende compounds BeS, BeSe, and BeTe. For comparison, we also perform calculations for the so-called Slater transition state [14], i.e. using half a core-hole. While it is often used in the literature to compute the energy of core-edges or core-level binding energy shifts [42], we are at present interested in its effect on the spectral shape. Partial core-holes are typically used in the literature in order to improve agreement with experiment as well as a means of reducing the supercell size [43, 44]. Here, we keep the number of atoms fixed, monitoring the effect of the reduced core-hole on the spectra. For all presented calculations, experimentally derived lattice constants are used [45]. The generalized gradient approximation (GGA) of Perdew et al. [46] is used as exchange-correlation functional.

In this work, we do not compute the self-energies to obtain the quasiparticle band structure based on the DFT results. Although all-electron $G_{0} W_{0}$ calculations have been demonstrated to reasonably describe semi-core states [47], state-of-the-art $G W$ calculations cannot reproduce the energetic positions of core states. Therefore, our theoretical spectra are aligned with respect to the edge peak for comparison with experiment. The zero is set at the calculated conduction band minimum with respect to the Be $1 s$ level. A Gaussian broadening of $0.5 \mathrm{eV}$ at full width at half maximum (FWHM) is applied to the calculated spectra for comparison with measurement data. Due to the anisotropy of the hexagonal wurtzite structure, we calculate an average over the two inequivalent crystal directions. 


\section{Experiment}

Experimental XAS spectra were recorded at beamline 8.0.1 of the Advanced Light Source, Lawrence Berkeley National Laboratory. The spectra were recorded in the partial fluorescence yield (PFY) mode (to reduce saturation effects) using the soft x-ray spectrometer of the permanently installed SXF end station [48] and integrating over an emission energy range of $99.5 \mathrm{eV}$ to $109.5 \mathrm{eV}$. The resolution of the beamline was set to be better than $0.25 \mathrm{eV}$. As samples, BeS powder with $99 \%$ purity and grain size up to $150 \mu \mathrm{m}$ (Alfa Aesar) and a BeO ceramic piece on a carbon tape support were used. The preparation of the BeSe and BeTe samples is described in [28]. Note that the BeS powder exhibits some surface oxide as will be discussed below.

\section{Results and discussion}

In figure 1, we show the XAS spectrum of the Be K-edge in wurtzite BeO. Theoretical $\mathrm{BSE}$ results (red line) and supercell calculations (SC) using the core-hole approximation for one hole (blue line) and half a hole (blue dotdashed line), are compared with the experimental data (black line). Comparison with the RPA spectrum (dotted gray line) demonstrates the dramatic effect of neglecting the influence of the core-hole. The vertical dashed zero line corresponds to the onset of the RPA spectrum and thus serves as a reference for bound core-exciton states in BSE. The result obtained by the PP BSE scheme [33] is also shown (thin green line). From figure 1, it is clear that the two different theoretical approaches, BSE and the supercell with a full core hole, produce an overall similar fine structure, closely following experiment (note that the weaker intensity contrast in the experimental spectrum is attributed to saturation effects in the partial fluorescence yield). Considering only half of a core hole in the SC, which corresponds to Slater's transition state, results in a reduction of intensity at the edge and a shift of the spectral features towards higher energy. As expected, RPA gives a completely different spectrum, not only in the region of the absorption onset, but throughout the entire energy range.

One difference between BSE and SC is a higher intensity of BSE at the coreedge, in line with earlier findings $[49,50,51]$. Likewise, at higher energies, the BSE spectrum exhibits corresponding features compared to the SC results. Such behaviour was found for the Li K-edge in the wide-gap lithium halides [49]. We find that the current FPLAPW BSE scheme gives similar spectra as the PP BSE calculations [33]. However, the latter spectrum appears more stretched at higher energies than our BSE and SC calculations. We assign this difference to the fact that the band structure underlying the PP BSE [33] calculations includes $G W$ self-energy corrections for the valence band region. Our SC results for 1.0 core-hole are similar to the ones obtained within the pseudopotential method by Gao et al. [36, 37].

In figure 2, we show the theoretical Be K-edge XAS spectrum for bulk zinc-blende BeS. It is compared with the experimental absorption spectra of a surface oxidized BeS 
powder. The surface oxidation is due to the fact that $\mathrm{BeS}$ is strongly hygroscopic, forming $\mathrm{BeO}$ and $\mathrm{H}_{2} \mathrm{~S}$ when exposed to air. Therefore, the BeS sample contains a clear $\mathrm{BeO}$ signal, which can be seen by comparing with the $\mathrm{BeO}$ spectrum in the previous figure 1. In experiment, the signal could be monitored for samples with different degrees of oxidation (not shown). While accurate measurements of the band gap is at present not available for BeS, GW calculations predict a direct gap of $7.4 \mathrm{eV}$ and an indirect gap of $4.7 \mathrm{eV}$ [38]. In contrast, $\mathrm{BeO}$ has a wide band gap of about $10.6 \mathrm{eV}[52,53]$. This means that the oxidized surface layer is transparent below the $\mathrm{BeO}$ absorption edge and we therefore attribute the prominent shoulder peak to BeS. The BSE and SC spectra of $\mathrm{BeS}$ are dominated by the core-edge feature, with a low (and relatively flat) intensity at higher energies. As in the case of $\mathrm{BeO}$, the two theoretical approaches produce a similar fine structure, with an enhanced excitonic peak when using BSE. The SC calculation with the Slater transition state reduces and shifts the intensity at the core-edge, which is completely gone in the RPA spectrum.

The Be $1 s$ absorption spectra for another two zinc-blende chalcogenides, BeSe and BeTe, are depicted in figure 3 and 4, respectively. In both cases, the experimental XAS spectra are those shown in [28], but plotted in a wider energy window. Once again, as observed above for $\mathrm{BeO}$ and $\mathrm{BeS}$, the two different theoretical approaches produce similar results. Only small differences are found in the fine structures, and the coreedge peaks are enhanced when using BSE. Our SC results are different from the ones obtained previously [28], because in the latter the core-hole approximation is not taken into account. Note that the experimental spectra also exhibit a small peak on the highenergy slope of the core-exciton resonance (approximately $1.5 \mathrm{eV}$ above the main line), which we ascribe to a minor contribution of oxidized Be at the surface of these samples. In line with the BSE and SC 1.0 hole calculations, the SC 0.5 hole and RPA spectra show a similar trend as for BeS in figure 2. Here, the reduced core-hole effect in the Slater transition state supercell calculations produces a similar shape of the core-edge peak as in the measurements.

\section{Discussion and conclusions}

Comparing the spectral features in the Be $1 s$ spectra obtained in the calculations, we notice that all cubic zinc-blende chalcogenides in figure 2-4 display the same overall spectral shape, with a strong core-exciton resonance, followed by a lower-intensity fine structure (the same behaviour is also inferred for BeS, see discussion above). This is different to the shape of the hexagonal wurtzite $\mathrm{BeO}$ spectrum, which, in addition to the core-exciton, also displays noticeable features at higher energies. This difference is retained for $\mathrm{BeO}$ in the zinc-blende structure, as shown by supercell core-hole calculations [37]. The spectral features can be understood by analyzing the excitations derived from solving the Bethe-Salpeter equation. For clarity, we show the individual excitations close to the core-edge in figure 5. In the case of $\mathrm{BeO}$ both the components parallel and orthogonal to the c-axis are shown. The strongest bound core-exciton 
is mainly of $s$-orbital character, and therefore does not contribute to the edge peak. Instead, we find that the core-edge peak in $\mathrm{BeO}$ is mainly due to a strongly bound exciton (in-plane) of about $1.5 \mathrm{eV}$ binding energy, as can be seen in the figure it is a degenerate state. The core-edge peak also contains two separate core-excitons (parallel to the wurtzite c-axis) of similar and smaller binding energy, respectively. In comparison, for the chalcogenides, the core-edge spectrum is composed of a multi-peak structure. Here, the most pronounced part at lowest energy correspond to bound core-excitons with the highest oscillator strength in the spectra, while the parts at higher energies consist of several excitations. The maximum core-exciton binding energies for BeS, BeSe, and BeTe, are 0.4, 0.3, and $0.25 \mathrm{eV}$, respectively. While the overall spectral shapes do not change much with $\mathbf{k}$ meshes higher than mentioned above, the binding energies require denser meshes to be fully converged. We estimate their accuracy to be within $0.1 \mathrm{eV}$.

Previously, it was found that BSE and supercell calculations using the core-hole approximation can give substantially differing results. For the Li K-edge in the Lihalides, it was demonstrated that it is necessary to use BSE in order to obtain a correct description of the fine structure $[49,50]$. For the $\mathrm{Mg} \mathrm{L}_{2,3}$-edge $(2 p)$ in $\mathrm{MgO}[50]$ and $\mathrm{Al}$ $\mathrm{L}_{2,3}$-edge in AlN polytypes [51], it was shown that BSE gives a better distribution of the overall spectral intensity. In the present case, for the Be K-edge in the oxygen-group compounds, we find a smaller difference between the two approaches. Still, an advantage of using BSE is the possibility of characterizing the excitons, which correspond to the dominant spectral features in the experimental XAS spectra.

Summarizing, we have used state-of-the-art first-principles calculations to investigate the Be $1 s$ near-edge x-ray absorption fine structure in the beryllium-oxygen group, including $\mathrm{BeO}$ and the chalcogenides BeS, BeSe, and BeTe. Measurements have been performed on $\mathrm{BeO}$ and $\mathrm{BeS}$ samples and have been combined with previously reported results on BeSe and BeTe. Theoretical spectra have been obtained by applying the Bethe-Salpeter equation using the open-source code exciting $[25,26]$ and by performing supercell calculations employing the core-hole approximation. In the present case, the two theoretical approaches give very similar results. All experimental spectra can be well described by taking surface oxidation of the material into account, especially in the case of the BeS powder. Note that while the BeS sample does not represent an ideal case due to the surface oxidation, it is nevertheless straightforward to identify its main characteristic, the core-edge peak. Further, theory predicts this marked edge peak, in line with the results for BeSe and BeTe. By solving the Bethe-Salpeter equation, we find that the core-edge peak in $\mathrm{BeO}$ is described by several relatively strongly bound core-excitons, while for the chalcogenide compounds the edge-peak consists of both bound excitons and other excitations. 


\section{Acknowledgments}

W.O. acknowledges funding from the Linköping Linnaeus Initiative for Novel Functional Materials (LiLi-NFM) supported by the Swedish Research Council (VR), and the VR grant number 691-2011-4426. Part of the calculations were carried out at the National Super Computer Centre (NSC) in Linköping University supported by the Swedish National Infrastructure for Computing (SNIC). This study was carried out within the framework of a Grant-in-Aid for Scientific Research (A) and a Grant-in-Aid for Scientific Research on Priority Areas "Nano Materials Science for Atomic Scale Modification 474" from the Ministry of Education, Culture, Sports, Science and Technology (MEXT) of Japan. Financial support by the European Theoretical Spectroscopy Facility (ETSF) is appreciated. We gratefully acknowledge the technical support of the ALS staff as well as funding by the Deutsche Forschungsgemeinschaft through SFB 410.

\section{References}

[1] Waag A, Fischer F, Shüll K, Baron T, Lugauer H-J, Lite Th, Zehnder U, Ossau W, Gerhard T, Keim M, Reuscher G and Landwehr G 1997 Appl. Phys. Lett. 70280

[2] Stöhr J 1992 NEXAFS spectroscopy (Berlin: Springer-Verlag)

[3] de Groot F and Kotani A 2008 Core level spectroscopy of solids (Boca Raton FL: CRC Press)

[4] Sham L J and Rice T M 1966 Phys. Rev. 144708

[5] Hanke W and Sham L J 1975 Phys. Rev. B 124501

[6] Hanke W and Sham L J 1979 Phys. Rev. Lett. 43387

[7] Hanke W and Sham L J 1980 Phys. Rev. B 214656

[8] Strinati G 1982 Phys. Rev. Lett. 491519

[9] Strinati G 1984 Phys. Rev. B 295718

[10] Onida G, Reining L and Rubio A 2002 Rev. Mod. Phys. 74601

[11] Hohenberg P and Kohn W 1964 Phys. Rev. 136 B864

[12] Kohn W and Sham L J 1965 Phys. Rev. 140 A1133

[13] von Barth U and Grossmann G 1982 Phys. Rev. B 255150

[14] Slater J C 1974 Quantum Theory of Molecules and Solids vol. 4 of International Series in Pure and Applied Physics 1st ed. (New York: McGraw-Hill)

[15] Rehr J J and Albers R C 2000 Rev. Mod. Phys. 72621

[16] Weijs P J W, Czyzyk M T, van Acker J F, Speier W, Goedkoop J B, van Leuken H, Hendrix H J M, de Groot R A, van der Laan G, Buschow K H J, Wiech G and Fuggle J C 1990 Phys. Rev. B 4111899

[17] Lerch P, Jarlborg T, Codazzi V, Loupias G and Flank A M 1992 Phys. Rev. B 4511481

[18] Mizoguchi T, Olovsson W, Ikeno H and Tanaka I 2010 Micron 41695

[19] Johansson B and Martensson M 1980 Phys. Rev. B 214427

[20] Weinert M, Davenport J W and Watson R E 1986 Phys. Rev. B 342971

[21] Olovsson W, Marten T, Holmström E, Johansson B and Abrikosov I A 2010 J. Electron Spec. Relat. Phenom. 178-9 88

[22] Jarlborg T, Barbiellini B, Lin H, Markiewicz R S and Bansil A 2011 Phys. Rev. B 84045109

[23] Rehr J J, Soininen J A and Shirley E L 2005 Physica Scripta T 115207

[24] Andersen O K 1975 Phys. Rev. B 123060

[25] http://exciting-code.org

[26] Sagmeister S and Ambrosch-Draxl C 2009 Phys. Chem. Chem. Phys. 114451

[27] Blaha P, Schwartz K, Madsen G, Kvasnicka D, and Luitz J 2001 WIEN2k An Augmented Plane 
Wave Plus Local Orbitals Program for Calculating Crystal Properties (Vienna: Schwartz K, Technische Universität Wien)

[28] Eich D, Fuchs O, Groh U, Weinhardt L, Fink R, Umbach E, Heske C, Fleszar A, Hanke W, Gross E K U, Bostedt C, v Buuren T, Franco N, Terminello L J, Keim M, Reuscher G, Lugauer H and Waag A 2006 Phys. Rev. B 73115212

[29] Käämbre T, Kikas A, Kooser K, Kisand V, Kirm M, Saar A, Nõmmiste E, Ivanov V, Pustarov V and Martinson I 2007 J. Electron Spectro. Relat. Phenom. 156-8 299

[30] Pustovarov V, Ivanov V, Kirm M, Kikas A, Kooser K, Käämbre T, Kruzhalov A and Zinin E 2007 Nuclear Instruments and Methods in Physics Research A $\mathbf{5 7 5} 172$

[31] Song H-F, Lei H-F and Tian E 2007 J. Phys.: Condens. Matter 19456209

[32] Bosak A, Schmalzl K, Krisch M, van Beek W and Kolobanov V 2008 Phys. Rev. B 77224303

[33] Soininen J A, Hämäläinen K, Caliebe W A, Kao C-C and Shirley E L 2001 J. Phys.: Condens. Matter 138039

[34] Shirley E L 1998 Phys. Rev. Lett. 80794

[35] Soininen J A and Shirley E L 2001 Phys. Rev. B 64165112

[36] Gao S-P, Pickard C J, Payne M C, Zhu J and Yuan J 2008 Phys. Rev. B 77115122

[37] Gao S-P 2010 Phys. Status Solidi B 2472190

[38] Fleszar A and Hanke W 2000 Phys. Rev. B 622466

[39] Rached D, Rabah M, Benkhettou N, Khenata R, Soudini B, Al-Douri Y and Baltache H 2006 Comp. Mat. Sci. $\mathbf{3 7} 292$

[40] Yadav P S, Yadav R K, Agrawal S and Agrawal B K 2007 Physica E 3679

[41] Puschnig P and Ambrosch-Draxl C 2002 Phys. Rev. B 66165105

[42] Göransson C, Olovsson W and Abrikosov I A 2005 Phys. Rev. B 72134203

[43] Hébert C, Luitz J and Schattschneider P 2003 Micron 34219

[44] Mizoguchi T, Tanaka I, Yoshiya M, Oba F, Ogasawara K and Adachi H 2000 Phys. Rev. B 61 2180

[45] Wyckoff R W G 1963 Crystal Structures 1 2nd ed. (New York: Interscience Publishers)

[46] Perdew J P, Burke K, and Ernzerhof M 1996 Phys. Rev. Lett. 773865

[47] Li X, Gomez-Abal R, Jiang H, Ambrosch-Draxl C and Scheffler M 2012 New J. Phys. 14023006

[48] Jia J J, Callcott T A, Yurkas J, Ellis A W, Himpsel F J, Samant M G, Stöhr J, Ederer D L, Carlisle J A, Hudson E A, Terminello L J, Shuh D K and Perera R C C 1995 Rev. Sci. Instrum. 661394

[49] Olovsson W, Tanaka I, Puschnig P and Ambrosch-Draxl C 2009 J. Phys.: Condens. Matter 21 104205

[50] Olovsson W, Tanaka I, Mizoguchi T, Puschnig P and Ambrosch-Draxl C 2009 Phys. Rev. B 79 041102R

[51] Olovsson W, Tanaka I, Mizoguchi T, Radtke G, Puschnig P and Ambrosch-Draxl C 2011 Phys. Rev. B 83195206

[52] Roessler D M and Walker W C 1969 J. Phys. Chem. Solids 30157

[53] Groh D, Pandey R, Sahariah M B, Amzallag E, Baraille I and Rérat M 2009 J. Phys. Chem. Solids $\mathbf{7 0} 789$ 


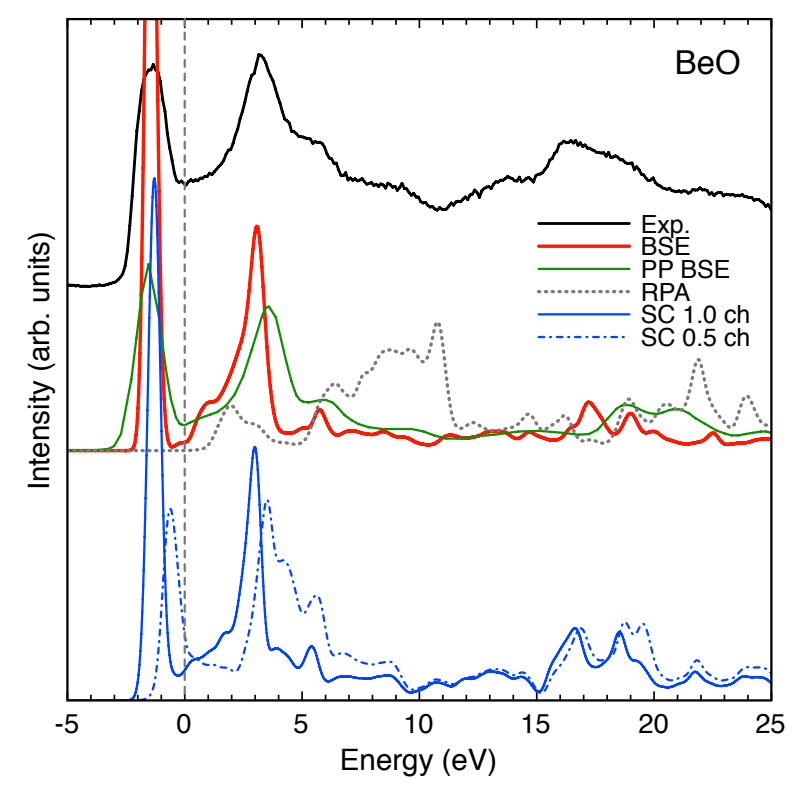

Figure 1: The Be K-edge absorption spectrum of wurtzite BeO. Top: experiment (black line), middle: BSE (red line), bottom: supercell calculations with one core hole (blue line) and half a core hole (blue dot-dashed line). The thin green line (middle) is taken from [33] and obtained by a BSE approach based on the pseudopotential approximation. The BSE and 1.0 core hole supercell calculations are aligned with respect to the experimental edge peak. The dotted gray line (middle) represents the RPA approach. The vertical dashed line corresponds to the onset of the RPA spectrum, i.e., conduction band minimum, also the reference for the bound core-exciton states in BSE.

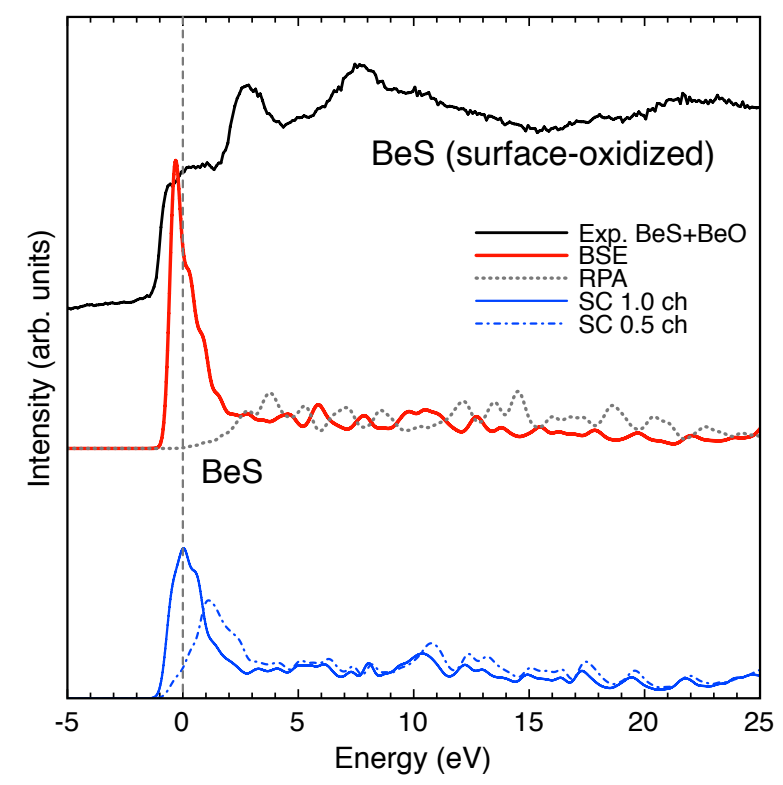

Figure 2: The Be K-edge XAS spectra comparing calculations for bulk zinc-blende BeS and experiments taken on surface-oxidized BeS powder. The notation is the same as in figure 1. 


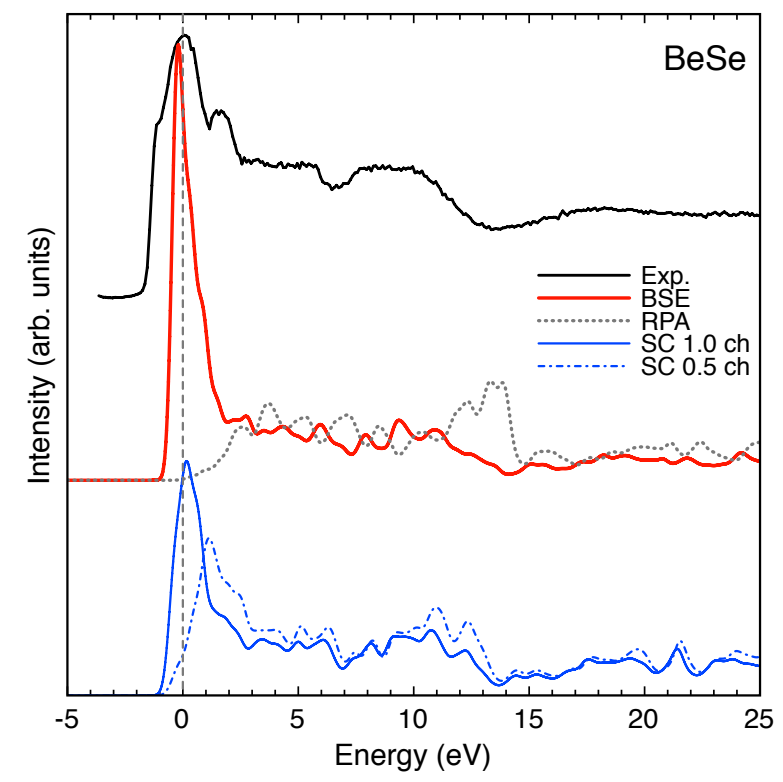

Figure 3: The Be K-edge in zinc-blende BeSe. Experimental data (black line, top) [28]. The notation is the same as in figure 1 .

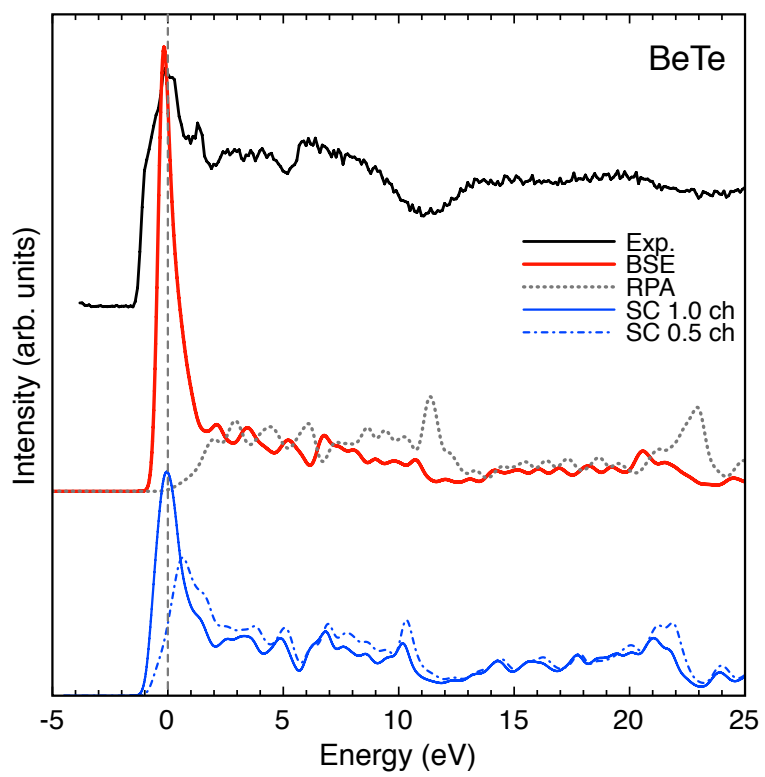

Figure 4: The Be K-edge in zinc-blende BeTe. Experimental data (black line, top) [28]. The notation is the same as in figure 1. 

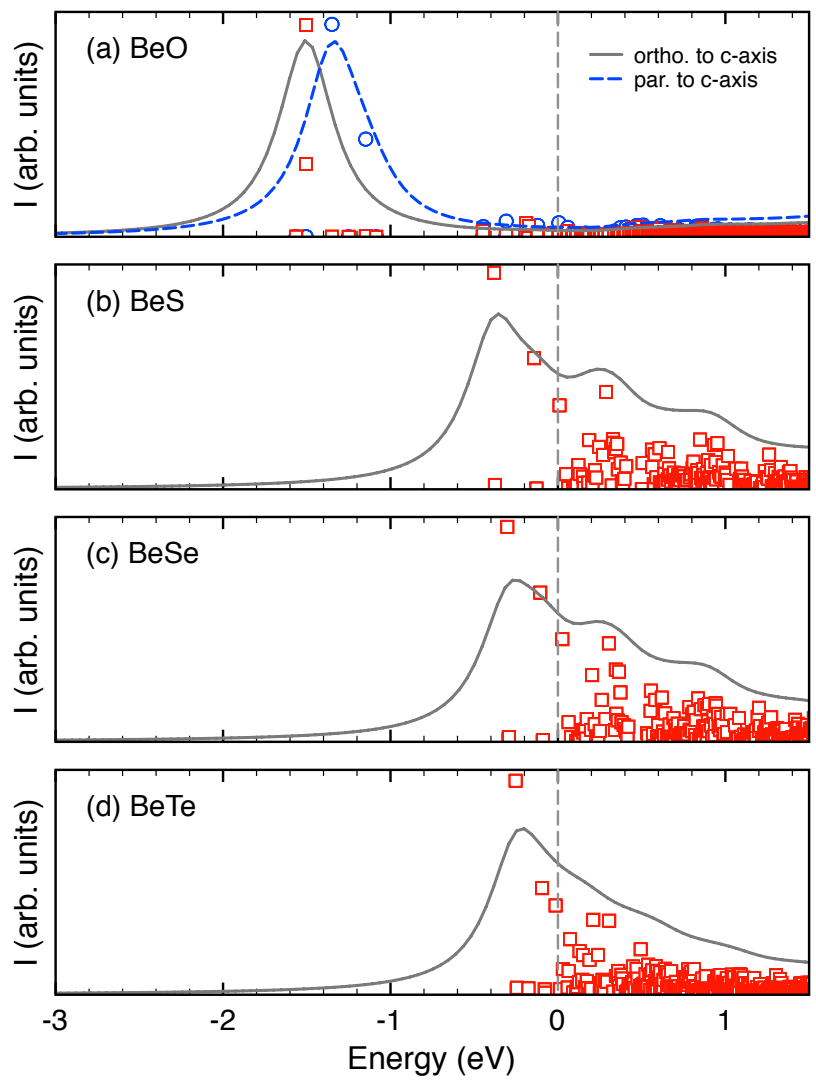

Figure 5: The Be K-edge excitations (symbols) and corresponding absorption spectrum (line) at the edge are shown for (a) BeO, (b) BeS, (c) BeSe and (d) BeTe. In the case of $\mathrm{BeO}$ both components orthogonal (red boxes, full line) and parallel (blue circles, dashed blue line) to the c-axis are shown. 